\title{
The lenticular galaxy NGC 3607: stellar population, metallicity, and ionised gas
}

\author{
M. G. Rickes, M. G. Pastoriza, and C. Bonatto
}

\author{
Universidade Federal do Rio Grande do Sul (UFRGS), Instituto de Física, Departamento de Astronomia, Brazil \\ e-mail: [maurogr;mgp; charles]@if.ufrgs.br
}

Received 11 December 2008 / Accepted 14 July 2009

ABSTRACT

\begin{abstract}
Aims. In this work we derive clues to the formation scenario and ionisation source of the lenticular galaxy NGC 3607 by means of metallicity gradients, stellar population, and emission lines properties.

Methods. We work with long-slit spectroscopy from which we (i) study the radial distribution of the equivalent widths of conspicuous metallic absorption features; (ii) make inferences about the star formation history (with a stellar population synthesis algorithm); and (iii) investigate the ionisation source responsible for a few strong emission lines.

Results. Negative radial gradients are observed for most of the absorption features of NGC 3607. Compared to the external parts, the central region has a deficiency of alpha elements and higher metallicity, which implies different star formation histories in both regions. At least three star formation episodes are detected, with ages within 1-13 Gyr. The dynamical mass and the $M g_{2}$ gradient slope are consistent with mergers being important contributors to the formation mechanism of NGC 3607, a scenario consistent with the star formation episodes.. Emission-line ratios indicate a LINER at the centre of NGC 3607. Contribution of hot, old stars to the gas ionisation outside the central region is detected.
\end{abstract}

Key words. galaxies: formation - galaxies: active - galaxies: individual: NGC 3607

\section{Introduction}

Significant efforts have been made in the past few years to understand the complex mechanisms associated with the formation and evolution of the early-type galaxies. Since the early 90 s, it has been established that ellipticals cannot be described as part of a one-parameter family and that many show unambiguous signatures of interaction with companions and the environment (van den Bergh 1990). These facts suggest that these galaxies may have experienced different star formation histories, with the present-day stellar populations differing in metallicity and/or age. In this sense, most early-type galaxies (or their nuclei) are not characterised by a single-aged stellar population. Instead, they are described better by a composition of single stellar populations (e.g. Worthey et al. 1992; Arimoto \& Yoshii 1987; Rickes et al. 2004; Rickes et al. 2008).

Observationally, clues to the star formation history of a galaxy can be found in the radial dependence of the stellar $[\alpha / \mathrm{Fe}]$ ratio, or more specifically the $\mathrm{Mg} / \mathrm{Fe}$ abundance ratio (Pipino et al. 2006). The $M g 2$ line-strength distribution in earlytype galaxies can vary considerably, with radial profiles ranging from essentially featureless to rather structured. In the latter case, changes in slope, possibly associated with kinematically decoupled cores, or anomalies in the stellar population, have been observed in some ellipticals (e.g. Carollo et al. 1993).

In this context, investigation of the spatial distribution of the stellar population and metallicity in samples of early-type galaxies may shed some light on these formation and evolution issues. With the above goals in mind, we have been conducting a systematic study of objects rich in interstellar medium, such as NGC 5044 (Rickes et al. 2004) and NGC 6868 (Rickes et al. 2008).
This work focus on the lenticular galaxy NGC 3607, which is the central and brightest member of a group that contains the galaxies NGC 3605 and NGC 3608 as well. Figure 1 shows a $15^{\prime} \times 15^{\prime} \mathrm{V}$ band image of NGC 3607 and companions, extracted from the Canadian Astronomy Data Centre (CADC) ${ }^{1}$.

The radial velocity of NGC 3607 is $\sim 960 \mathrm{~km} \mathrm{~s}^{-1}$ (Ferguson \& Sandage 1990), which puts it at a distance of $\sim 12.8 \mathrm{Mpc}$ (with $\left.H_{0}=75 \mathrm{~km} \mathrm{~s}^{-1} \mathrm{Mpc}^{-1}\right)$. At such a distance, $1^{\prime \prime}$ corresponds to about $0.062 \mathrm{kpc}$. NGC 3607 is a LINER with X-ray emission (Terashima et al. 2002), and discrete sources in the host galaxy are the probable origin of the extended hard X-ray emission. The hot gas in this elliptical galaxy has been analysed by Matsushita et al. (2000). NGC 3607 is another case where the dust and ionised gas distribute as a small asymmetric disk. The dust absorption is stronger in the northeast direction, while the ionised gas emission is more significant towards the southwest (Ferrari et al. 1999).

The goal of this paper is to investigate the stellar population, metallicity distribution, and the ionised gas in NGC 3607, which are important parameters for understanding the formation and evolution of this galaxy.

This paper is organised as follows: Sect. 2 presents the observations and data reduction; Sect. 3 deals with the equivalent width analysis of the absorption lines and their radial dependence; Sect. 4 describes the stellar population synthesis, whose results are discussed in Sect. 5; Sect. 6 presents an analysis of the emission lines and the nature of the ionisation source. Finally, Sect. 7 offers a general discussion of the results and conclusions.

\footnotetext{
${ }^{1}$ http://cadcwww.dao.nrc.ca/
} 


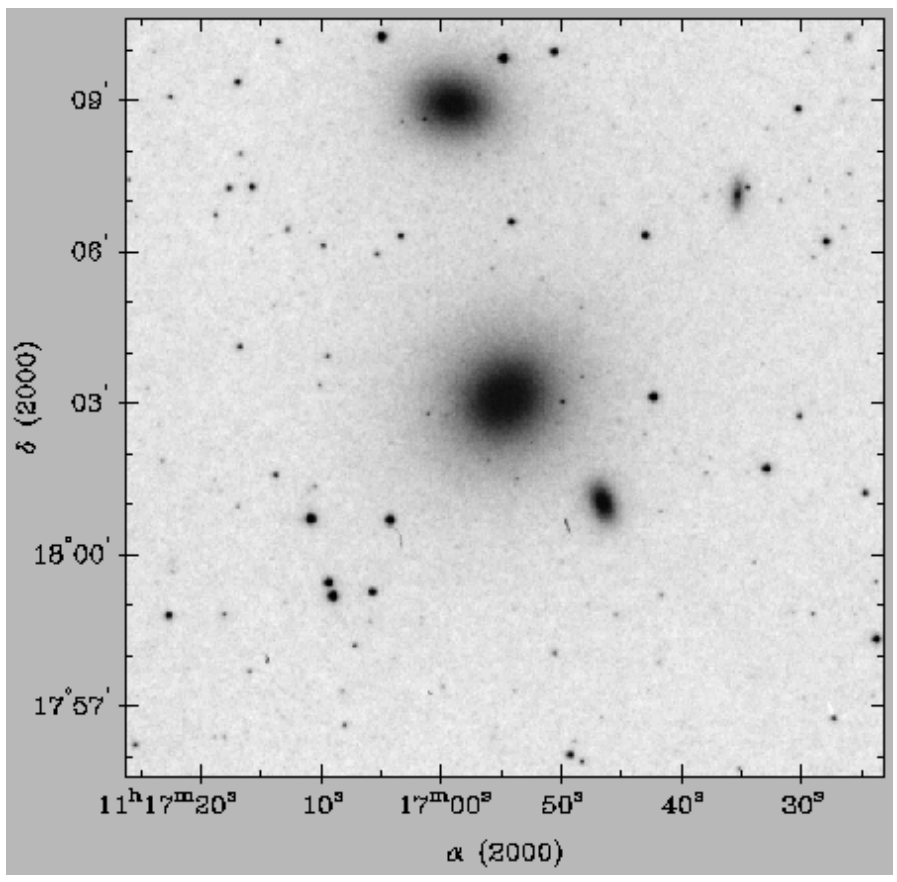

Fig. 1. $V$ band image covering the field $15^{\prime} \times 15^{\prime}$ centred on NGC 3607 . Image orientation: north to the top and east to the left.

Table 1. General data on NGC 3607.

\begin{tabular}{ll}
\hline \hline Parameter & Value \\
\hline$\alpha(\mathrm{J} 2000)$ & $11^{\mathrm{h}} 16^{\mathrm{m}} 54.6^{\mathrm{s}}$ \\
$\delta(\mathrm{J} 2000)$ & $+18^{\circ} 03^{\prime} 07^{\prime \prime}$ \\
$M_{B}$ & -21.92 \\
$B$ & 10.8 \\
$E(B-V)$ & 0.070 \\
$L_{\mathrm{H} \alpha+[\mathrm{NII}]}$ & $1.28 \times 10^{41} \mathrm{erg} \mathrm{s}^{-1}$ \\
Heliocentric radial velocity & $960 \pm 20 \mathrm{~km} \mathrm{~s}^{-1}$ \\
Distance & $12.8 \mathrm{Mpc}$ \\
Redshift & $0.003202 \pm 0.000067$ \\
Diameters & $4.9^{\prime} \times 2.5^{\prime}$ \\
Effective radius ${ }^{\dagger}$ & $43.4^{\prime \prime}$ \\
\hline
\end{tabular}

Note. Data obtained at the NASA/IPAC Extragalactic Database (NED) which is operated by the Jet Propulsion Laboratory, California Institute of Technology, under contract with the National Aeronautics and Space Administration. ( $\dagger$ ) $R_{\mathrm{e}}$ from Annibali et al. (2007).

\section{Observations and spectra extraction}

In the present paper we study the star formation history, metallicity distribution, and emission-gas properties of NGC 3607. Basically, we work with long-slit spectroscopy and a star formation synthesis algorithm, following similar methods to those applied to NGC 6868 and NGC 5903 (Rickes et al. 2008). The general parameters of the bright S0 galaxy NGC 3607 are given in Table 1.

The observations were obtained with the ESO $3.6 \mathrm{~m}$ telescope at La Silla, Chile, equipped with EFOSC1. The spectra cover the range $\lambda \lambda 5100-6800 \AA$ with a $3.6 \AA /$ pixel resolution. The spatial scale of the observational configuration is $0.6^{\prime \prime}$ pixel $^{-1}$; the slit length was $3.1^{\prime}$, while its width was fixed at $1.5^{\prime \prime}$, corresponding approximately to the average seeing; two 2-dimensional spectra were obtained with the same exposition time (approximately $45 \mathrm{~min}$ ) and position angle $\mathrm{PA}=301^{\circ}$. In

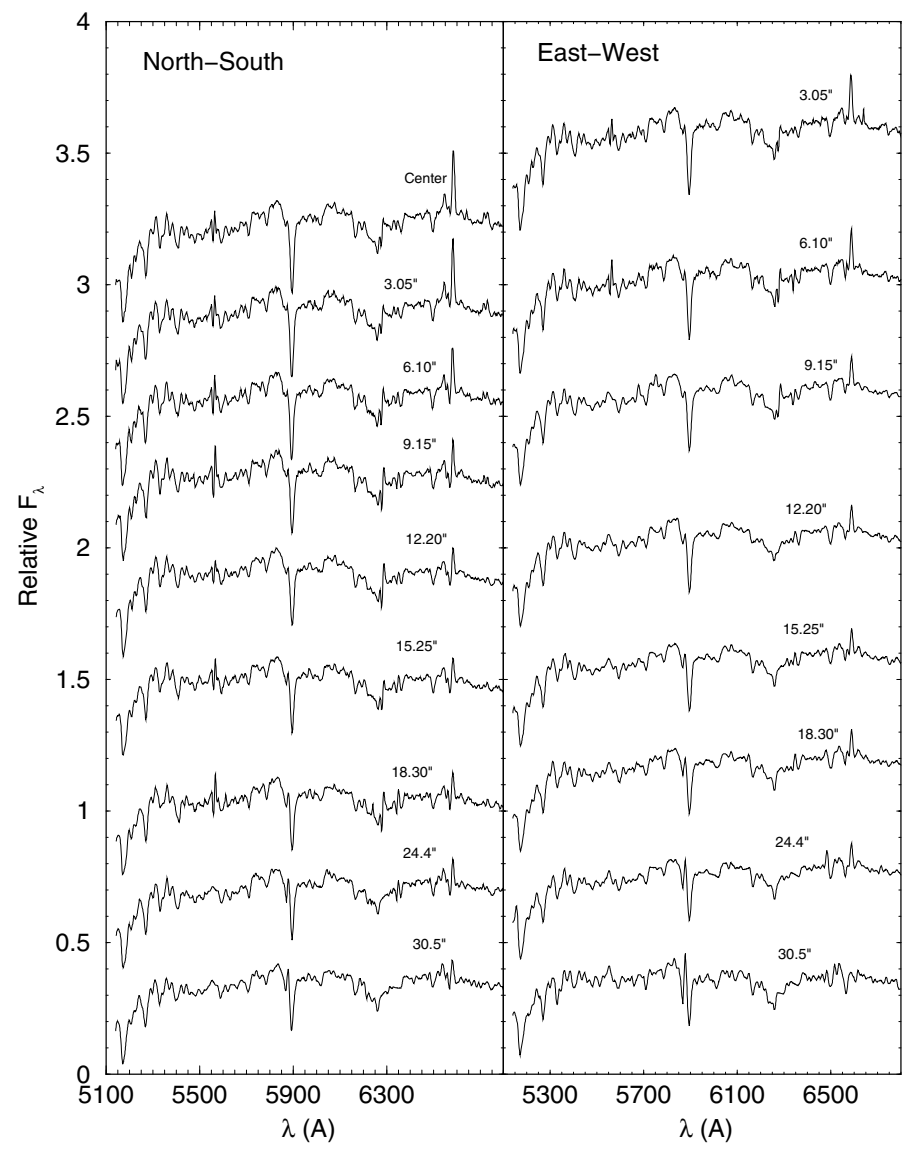

Fig. 2. Spatial extractions for the SW (left panel) and NE (right panel) directions normalised at $\lambda 5870 \AA$. The distance to the galaxy centre is indicated on each spectrum. Except for the bottom ones, the spectra have been shifted by an arbitrary constant, for clarity purposes.

all cases, the extraction area was fixed at $4.57^{\prime \prime 2}$, which corresponds to $\approx 0.02 \mathrm{kpc}^{2}$.

The standard star $H R 7333$ was observed for flux and velocity calibrations. We point out that the average $F W H M$ of the absorption features in the spectrum of $H R 7333$ is between 8 and $10 \AA$, similar to that of the Lick/IDS system.

The spectra were corrected for the radial velocity $V_{\mathrm{r}}=$ $960 \mathrm{~km} \mathrm{~s}^{-1}$ and the foreground Galactic extinction $A_{V}=0.035$ (Table 1). The corrected spectra, normalised at $\lambda 5870 \AA$, are shown in Fig. 2, separately for the northeast and southwest directions on the galaxy.

\section{Radial properties of absorption features}

Important information on the chemical abundance and stellar population of a galaxy can be obtained from the analysis of the equivalent widths of a set of selected absorption lines and continuum fluxes (e.g. Rickes et al. 2008; Rickes et al. 2004, and references therein). Here we work with the continuum tracing and spectral windows for the absorption features defined by the Lick system (Faber et al. 1985).

Because of the somewhat restricted coverage of our NGC 3607 spectra (Sect. 2), the available absorption features are

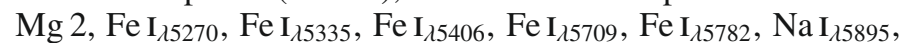
and $\mathrm{TiO}_{\mathrm{II}}{ }_{26237}$. Continuum points within the same spectral region are $\lambda_{5300}, \lambda_{5546}, \lambda_{5650}, \lambda_{5800}, \lambda_{5870}, \lambda_{6173}, \lambda_{6620}$, and $\lambda_{6640}$.

Some early-type galaxies have been shown to present systematic spatial variations on the line strength of the indices $\mathrm{Mg} 2$ 
Table 2. Equivalent widths measured in the spectra of NGC 3607.

\begin{tabular}{|c|c|c|c|c|c|c|c|c|c|c|}
\hline $\begin{array}{l}R \\
\left({ }^{\prime \prime}\right) \\
\end{array}$ & $R / R_{\mathrm{e}}$ & $\begin{array}{c}M g_{2} \text { index } \\
(\mathrm{mag})\end{array}$ & $\begin{array}{c}M g_{2 \lambda 5176} \\
(\AA) \\
\end{array}$ & $\begin{array}{c}\mathrm{FeI}_{\lambda 5270} \\
(\AA) \\
\end{array}$ & $\begin{array}{c}\mathrm{FeI}_{\lambda 5335} \\
(\AA) \\
\end{array}$ & $\begin{array}{c}\mathrm{FeI}_{\lambda 5406} \\
(\AA) \\
\end{array}$ & $\begin{array}{c}\mathrm{FeI}_{\lambda 5709} \\
(\AA)\end{array}$ & $\begin{array}{c}\mathrm{FeI}_{\lambda 5782} \\
(\AA) \\
\end{array}$ & $\begin{array}{c}\mathrm{NaI}_{\lambda 5895} \\
(\AA) \\
\end{array}$ & $\begin{array}{c}\mathrm{TiO}_{\mathrm{II} \lambda 6237} \\
(\AA)\end{array}$ \\
\hline 0.00 & 0.00 & $0.17 \pm 0.01$ & $6.10 \pm 0.22$ & $3.60 \pm 0.17$ & $3.75 \pm 0.15$ & $2.27 \pm 0.04$ & $1.33 \pm 0.02$ & $1.01 \pm 0.05$ & $5.03 \pm 0.21$ & $6.61 \pm 0.33$ \\
\hline $3.05 \mathrm{~S}$ & 0.07 & $0.16 \pm 0.01$ & $6.03 \pm 0.25$ & $3.58 \pm 0.35$ & $3.29 \pm 0.26$ & $2.35 \pm 0.09$ & $1.34 \pm 0.04$ & $1.06 \pm 0.01$ & $5.36 \pm 0.22$ & $6.42 \pm 0.32$ \\
\hline $6.10 \mathrm{~S}$ & 0.14 & $0.16 \pm 0.01$ & $5.78 \pm 0.35$ & $3.46 \pm 0.34$ & $3.13 \pm 0.52$ & $2.38 \pm 0.03$ & $1.43 \pm 0.06$ & $1.15 \pm 0.01$ & $4.76 \pm 0.25$ & $6.41 \pm 0.35$ \\
\hline $9.15 \mathrm{~S}$ & 0.21 & $0.15 \pm 0.01$ & $5.49 \pm 0.38$ & $3.45 \pm 0.28$ & $3.38 \pm 0.20$ & $2.08 \pm 0.11$ & $1.30 \pm 0.03$ & $1.29 \pm 0.03$ & $4.72 \pm 0.30$ & $6.54 \pm 0.33$ \\
\hline $12.20 \mathrm{~S}$ & 0.28 & $0.15 \pm 0.01$ & $5.56 \pm 0.38$ & $3.08 \pm 0.24$ & $3.07 \pm 0.29$ & $2.02 \pm 0.15$ & $1.02 \pm 0.10$ & $1.26 \pm 0.08$ & $4.04 \pm 0.27$ & $5.64 \pm 0.35$ \\
\hline $15.25 \mathrm{~S}$ & 0.35 & $0.14 \pm 0.01$ & $4.97 \pm 0.26$ & $2.80 \pm 0.48$ & $2.76 \pm 0.16$ & $2.35 \pm 0.06$ & $1.08 \pm 0.08$ & $1.12 \pm 0.02$ & $4.00 \pm 0.24$ & $5.65 \pm$ \\
\hline $18.30 \mathrm{~S}$ & 0.42 & $0.14 \pm 0.01$ & $4.95 \pm 0.40$ & $2.80 \pm 0.56$ & $2.73 \pm 0.30$ & $2.27 \pm 0.07$ & $1.06 \pm 0.11$ & $1.01 \pm 0.07$ & $3.99 \pm 0.31$ & $4.85 \pm 0.34$ \\
\hline $24.40 \mathrm{~S}$ & 0.56 & $0.13 \pm 0.01$ & $4.89 \pm 0.53$ & $3.14 \pm 0.49$ & $2.76 \pm 0.27$ & $1.86 \pm 0.14$ & $1.05 \pm 0.11$ & $1.33 \pm 0.13$ & $3.93 \pm 0.32$ & $5.63 \pm 0.37$ \\
\hline $30.50 \mathrm{~S}$ & 0.70 & $0.14 \pm 0.01$ & $4.92 \pm 0.62$ & $3.21 \pm 0.63$ & $2.65 \pm 0.57$ & $1.79 \pm 0.18$ & $1.23 \pm 0.12$ & $0.92 \pm 0.02$ & $3.43 \pm 0.32$ & $5.35 \pm 0.38$ \\
\hline $3.05 \mathrm{~N}$ & 0.07 & $0.16 \pm 0.01$ & $6.03 \pm 0.20$ & $3.64 \pm 0.33$ & $3.43 \pm 0.17$ & $2.28 \pm 0.04$ & $1.31 \pm 0.04$ & $1.00 \pm 0.02$ & $5.05 \pm 0.23$ & $6.38 \pm 0.33$ \\
\hline $6.10 \mathrm{~N}$ & 0.14 & $0.16 \pm 0.01$ & $5.89 \pm 0.28$ & $3.54 \pm 0.37$ & $3.34 \pm 0.26$ & $2.26 \pm 0.15$ & $1.22 \pm 0.05$ & $1.03 \pm 0.03$ & $4.67 \pm 0.30$ & $6.36 \pm 0.35$ \\
\hline $9.15 \mathrm{~N}$ & 0.21 & $0.15 \pm 0.01$ & $5.52 \pm 0.08$ & $3.45 \pm 0.24$ & $3.15 \pm 0.32$ & $2.21 \pm 0.16$ & $1.30 \pm 0.04$ & $1.19 \pm 0.02$ & $4.96 \pm 0.27$ & $6.40 \pm 0.31$ \\
\hline $12.20 \mathrm{~N}$ & 0.28 & $0.14 \pm 0.01$ & $5.39 \pm 0.28$ & $3.18 \pm 0.27$ & $3.00 \pm 0.24$ & $2.06 \pm 0.09$ & $1.07 \pm 0.07$ & $1.18 \pm 0.02$ & $4.56 \pm 0.31$ & $5.55 \pm 0.33$ \\
\hline $15.25 \mathrm{~N}$ & 0.35 & $0.14 \pm 0.01$ & $5.09 \pm 0.16$ & $2.84 \pm 0.51$ & $2.55 \pm 0.24$ & $2.22 \pm 0.10$ & $1.11 \pm 0.12$ & $1.03 \pm 0.05$ & $4.08 \pm 0.25$ & $5.46 \pm 0.38$ \\
\hline $18.30 \mathrm{~N}$ & 0.42 & $0.15 \pm 0.01$ & $5.00 \pm 0.30$ & $2.81 \pm 0.45$ & $2.74 \pm 0.13$ & $2.21 \pm 0.10$ & $1.03 \pm 0.14$ & $0.95 \pm 0.02$ & $4.02 \pm 0.27$ & $4.78 \pm 0.35$ \\
\hline $24.40 \mathrm{~N}$ & 0.56 & $0.14 \pm 0.01$ & $4.93 \pm 0.23$ & $2.93 \pm 0.50$ & $2.74 \pm 0.26$ & $1.93 \pm 0.13$ & $1.02 \pm 0.05$ & $1.29 \pm 0.05$ & $3.89 \pm 0.30$ & $5.65 \pm 0.37$ \\
\hline $30.50 \mathrm{~N}$ & 0.70 & $0.13 \pm 0.01$ & $4.89 \pm 0.12$ & $3.10 \pm 0.60$ & $2.59 \pm 0.46$ & $1.82 \pm 0.23$ & $1.19 \pm 0.10$ & $0.98 \pm 0.01$ & $3.55 \pm 0.31$ & $5.47 \pm 0.35$ \\
\hline
\end{tabular}

Note. $E W$ of $\mathrm{Mg}_{2}$ index is in mag. All spectra have been extracted within $4.57\left({ }^{\prime \prime}\right)^{2} \approx 0.02 \mathrm{kpc}^{2}$. The EWs are corrected for velocity dispersion, and the errors are the standard deviation of the average of three different measurements.

and $\mathrm{Fe} \mathrm{I}$, either from the centre to the external regions or limited to the central region (e.g. Gonzáles 1993; Carollo \& Danziger 1994; Carollo et al. 1993; Davis et al. 1993; Fisher \& Illingworth 1995; Fisher \& Illingworth 1996).

The above arguments suggest changes in some fundamental properties of the underlying stellar population. To investigate the stellar population and metallicity gradient in NGC 3607, we first analysed the spatial distribution of metal-line strengths, and then applied a stellar population analysis.

Strong absorption lines of neutral iron and sodium, as well as $\mathrm{Mg} 2$ and TiO bands, are present in the spectra of NGC 3607 (Fig. 2). Particularly conspicuous features are the $\operatorname{Mg} 2_{\lambda 5176}$, Fe $\mathrm{I}_{\lambda 5270}, \mathrm{Fe}_{\lambda 5335}, \mathrm{Fe}_{\lambda 5406}, \mathrm{Fe}_{\mathrm{I}} \mathrm{I}_{5709}, \mathrm{Fe}_{\lambda 5782}, \mathrm{Na}_{\lambda 5895}$, and $\mathrm{TiO}_{21}$ 6237. These absorption features present a strong dependence with the age and metallicity of the underlying stellar population (e.g Bica \& Alloin 1986; Rickes et al. 2004; Rickes et al. 2008).

Each equivalent width $(E W)$ was measured three times, allowing for the uncertainties in the continuum level definition from noise in the spectra. This procedure allowed us to estimate the average value and corresponding standard deviation from the average for each measurement.

Subsequently, the observed values of $E W$ were corrected for line broadening due to the stellar velocity dispersion. We followed the same procedure as described in Rickes et al. (2008). The spectrum of the G giant star $H R 7333$, assumed to have zero intrinsic velocity, was broadened with a series of Gaussian filters with velocity dispersion $\sigma$ varying from 0 to $300 \mathrm{~km} \mathrm{~s}^{-1}$ in steps of $10 \mathrm{~km} \mathrm{~s}^{-1}$. For each absorption feature considered, we calculated an empirical correction index $C_{\sigma}$, so that $C_{\sigma}(E W)=\frac{E W(0)}{E W(\sigma)}$, where $E W(0)$ and $E W(\sigma)$ are the observed and broadened equivalent widths, respectively. We calculated the correction index for each extracted spectrum using the $\sigma$ values defined by Caon et al. (2000).

The EWs measured in the spectra of NGC 3607, corrected for the velocity dispersion, are given in Table 2. Except for the
Mg2 index, which is measured in magnitude, the remaining EWs are given in $\AA$.

The corrected EWs of NGC 3607 (Table 2) are shown as a function of the distance to the galaxy centre in Fig. 3. Except for $\mathrm{Fe}_{\lambda} \mathrm{I}_{\lambda 782}$, the remaining absorption features present significant variations with distance to the centre. In particular, the $\mathrm{Mg} 2$ band clearly shows a negative gradient, i.e. increasing towards the centre of the galaxy. Evidence of similar negative gradients are seen in the other Fe I features.

Similar gradient slopes in the Mg 2 index and the Fe I features suggest the same chemical enhancement process for these elements. Furthermore, as shown in Fig. 4, the $E W$ of $\mathrm{Mg} 2$ correlates both with $E W\left(\mathrm{Fe}_{\lambda 5270}\right)$ and $E W\left(\mathrm{Fe}_{\lambda 5335}\right)$, which is consistent with the same enrichment process acting on $\mathrm{Mg}$ and $\mathrm{Fe}$ in NGC 3607.

When the gravitational potential is strong enough to retain the gas ejected by supernovae events and massive stars winds, it eventually migrates to the galaxy's central regions. Thus, it should be expected that subsequent generations of stars will be more metal-rich at the centre than in the external parts. Consequently, a negative radial metallicity gradient should be established as a mass-dependent parameter. In this scenario, a galaxy formed essentially through a monolithic collapse, i.e. with a small contribution of mergers, is expected to present a well-defined correlation between the metallicity gradient (Larson 1974; Ogando et al. 2005) and total mass (Carollo et al. 1993).

The above issue was investigated by Carollo et al. (1993), who studied the dependence of the gradient $\mathrm{dMg}_{2} / \mathrm{dlog} r$ with galaxy mass in a sample of 42 elliptical galaxies. They detected a conspicuous correlation for masses lower than $\sim 10^{11} M_{\odot}$, followed by a saturation for higher masses. Different formation mechanisms, such as a less dissipative collapse and/or merger of smaller galaxies, are invoked to explain the lack of an $M g_{2}$ gradient slope to galaxy mass correlation for the high-galaxy mass range (e.g. Carollo et al. 1993). In addition, the correlation between $\mathrm{dMg}_{2} / \mathrm{dlog} r$ with velocity dispersion $(\sigma)-$ confined to 

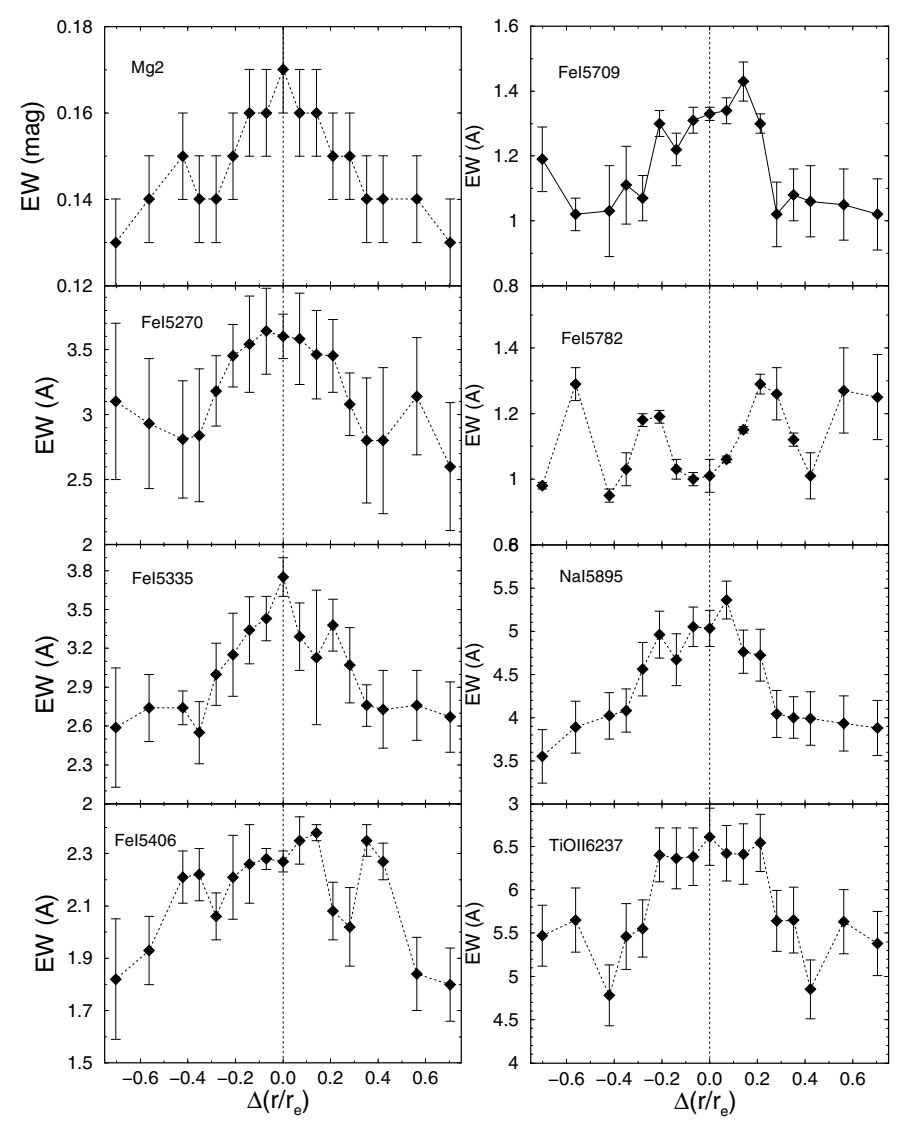

Fig. 3. Radial distribution of the absorption features in NGC 3607. The radial scale is given in terms of the effective radius, $R_{\mathrm{e}}=43.4^{\prime \prime}$, with negative values in the north direction.

the low-mass galaxies found by Ogando et al. (2005) - suggest that only galaxies below some limiting $\sigma$ have been formed by collapse, whereas the massive ones were formed predominantly by mergers.

To check how NGC 3607 fits in the metallicity gradient vs. galaxy mass picture of Carollo et al. (1993), we first estimated the galaxy's dynamical mass with the approximation of Poveda \& Allen (1975), $M_{\mathrm{tot}}=0.9 \sigma^{2} R_{\mathrm{e}} / G=3.34 \times 10^{8} M_{\odot}$, where $G$ is the gravitational constant, $R_{\mathrm{e}}=28.53^{\prime \prime}$ the effective radius, and $\sigma=225 \mathrm{~km} \mathrm{~s}^{-1}$ the central stellar velocity dispersion. The dynamical mass computed for NGC 3607 is $M=(1.6 \pm 0.3) \times 10^{11} M_{\odot}$ which, together with the $\mathrm{Mg} 2$ index gradient slope $\mathrm{d} M g_{2} / \mathrm{dlog} r=-0.02$, puts this galaxy in the merger-dominated region (see Fig. 11 of Carollo et al. 1993).

\section{Stellar population synthesis}

In this section we investigate the star formation history and derive properties of the stellar population of NGC 3607 by means of the stellar population synthesis method of Bica (1988). As population templates we use the synthetic star cluster spectra of Bruzual \& Charlot $(2003)^{2}$.

As a first approach we tested large bases with templates aged between $1 \mathrm{Myr}$ and $13 \mathrm{Gyr}$, but we found that the flux contribution of the components younger than a few $10^{2}$ Myr is statistically meaningless. Thus, for simplicity we built a spectral base

2 Built with a Salpeter (1955) Initial Mass Function.

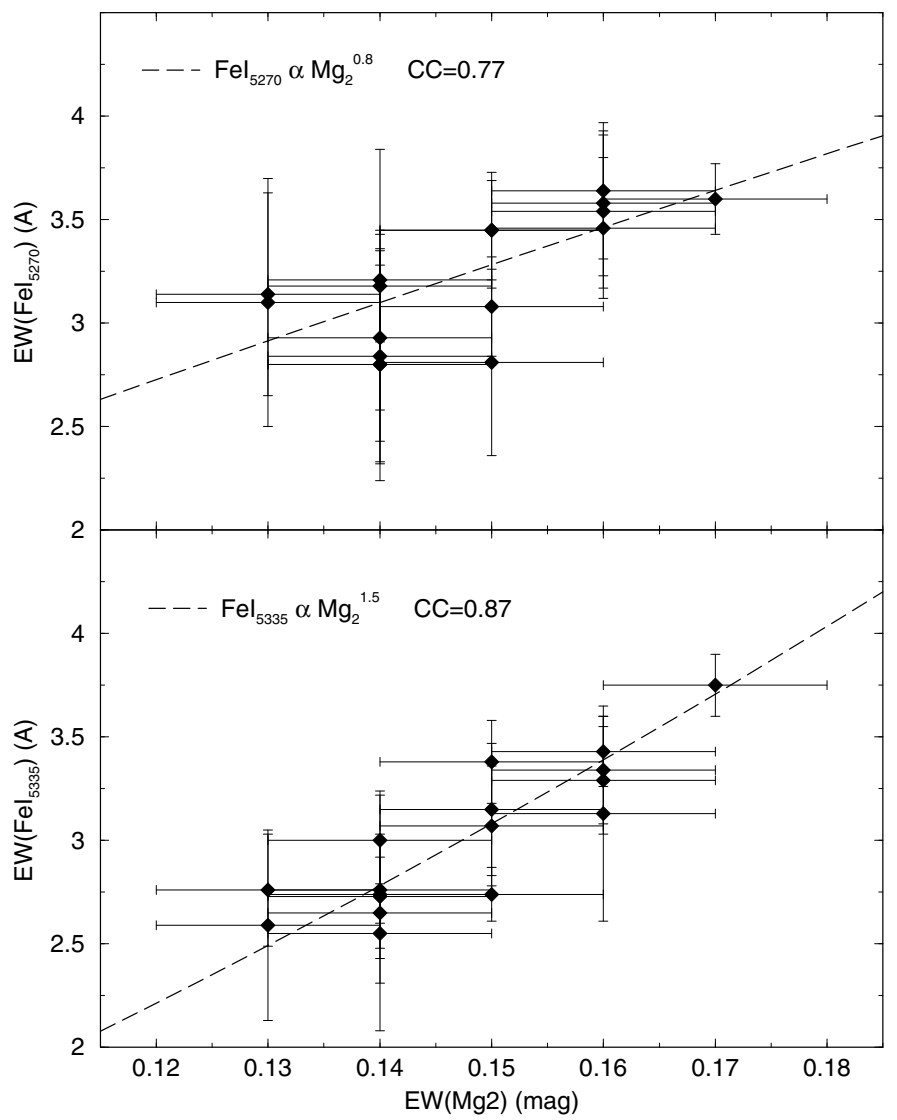

Fig. 4. Correlations among the EWs of selected absorption features measured in NGC 3607. The correlation coefficients (CC) of the fits are indicated.

containing 7 templates. After several tests we verified that the base that best reproduces the observed spectra, without redundancy, is the one with the ages 1,5 , and $13 \mathrm{Gyr}$, together with the metallicities $Z=0.008,0.02$, and 0.05 . These values are taken as representative of the sub-solar, solar and above-solar metallicity ranges (e.g. Rickes et al. 2008). The distribution of templates in the age-metallicity plane is shown in Table 3 . We note that the working base is rather restricted in components to minimise potential age/metallicity degeneracies (e.g. Rickes et al. 2008) due to the relatively small number of absorption features available in our spectra of NGC 3607 (Fig. 2).

We now compare the present stellar population synthesis results with previous works. Recently, Annibali et al. (2007) have studied the underlying stellar population of a large sample of early-type galaxies, among them NGC 3607, by means of the $\mathrm{H} \beta$, Iron, and Mgb age and metallicity indicators. They report an average stellar population age of $3.1 \mathrm{Gyr}$ for this galaxy, while we found an average age of $8.8 \mathrm{Gyr}$, based on the the central and external region values, a value that is more consistent for an SO galaxy. This discrepancy could stem from the different methodologies used to obtain the age of NGC 3607. Interestingly, we note that this does not occur with other galaxies of Annibali et al. (2007), which were studied by us following the same techniques as in the present paper. For NGC 5044 and NGC 6868 (Rickes et al. 2004, 2008), we obtained the average ages of 13 and $9.8 \mathrm{Gyr}$, respectively, which are very close to the 14.2 and 9.2 Gyr found by Annibali et al. (2007).

The stellar population synthesis algorithm directly provides the flux fractions (relative to the flux at $\lambda 5870 \AA$ ) that each age 
Table 3. Age and metallicity components.

\begin{tabular}{rccc}
\hline \hline & $Z=0.05$ & $Z=0.02$ & $Z=0.008$ \\
\hline $13 \mathrm{Gyr}$ & A1 & A2 & A3 \\
$5 \mathrm{Gyr}$ & $\mathrm{B} 1$ & $\mathrm{~B} 2$ & B3 \\
$1 \mathrm{Gyr}$ & & $\mathrm{C} 2$ & \\
\hline
\end{tabular}

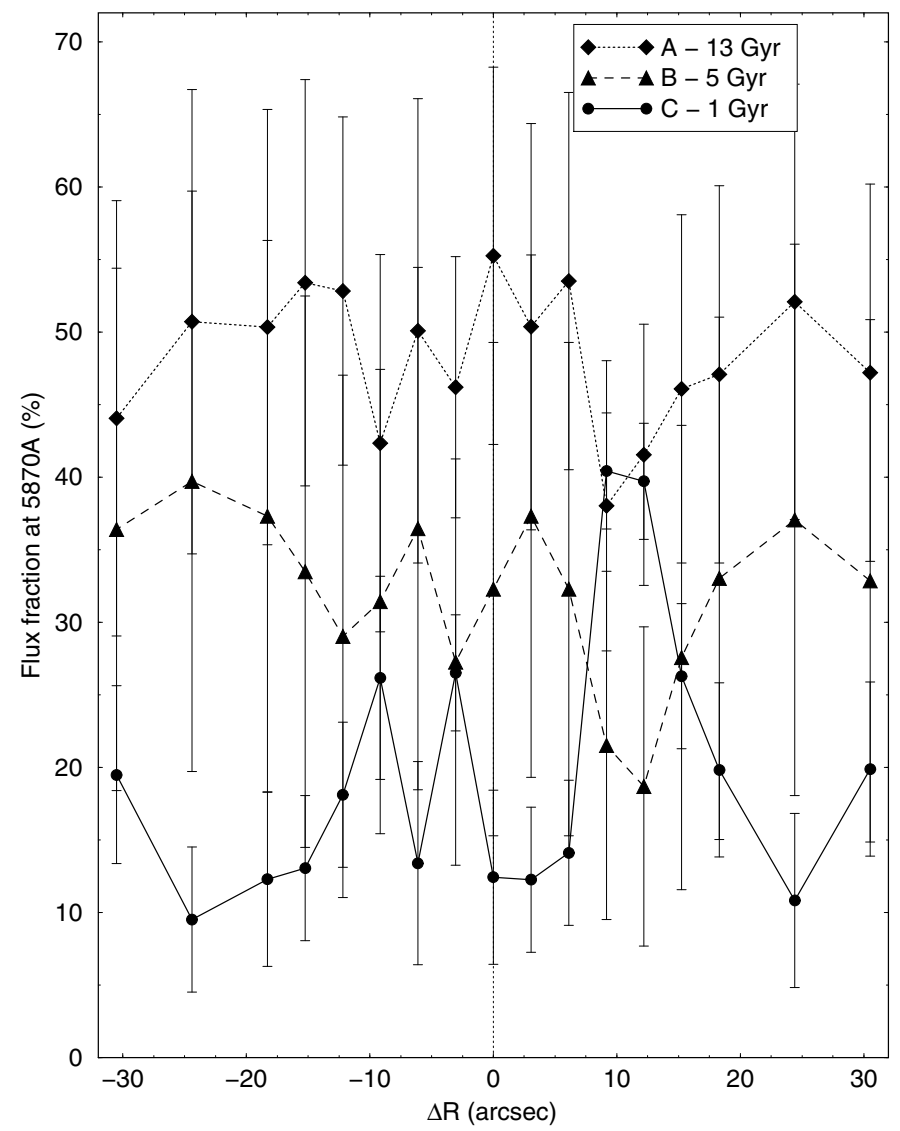

Fig. 5. Flux-fraction contribution at $5870 \AA$ of the stellar-population templates to the spectra of NGC 3607. Negative values in the north direction.

and metallicity template contributes to the observed spectrum. The sum of the template spectra, according to the individual flux fractions, should be representative of the stellar population contribution to the observed spectrum. A detailed description of the algorithm is provided in Rickes et al. (2004).

Results of the stellar population synthesis are summarised in Fig. 5, which shows the 1, 5, and $13 \mathrm{Gyr}$ component contributions to the flux at $5870 \AA$, along most of the galaxy's radial extent. The 5 and 13 Gyr curves in Fig. 5 correspond to the sum over the three metallicity components. In all cases the solar metallicity is the dominant component with a contribution higher than $70 \%$, while the $Z=0.05$ component contributes with $\approx 10 \%$, and the $Z=0.008$ with the remaining $\approx 20 \%$. Within uncertainties, the 5 and 13 Gyr components dominate the spectra, from the centre to the external parts. The error bars in Fig. 5 are the standard deviation from the average of all possible solutions for each stellar population template.

As a caveat we note that our observed spectra of NGC 3607 cover a relatively short spectral range. Thus, they contain a restricted number of indices sensitive to age and metallicity that, in principle, could provide a small number of constraints for the

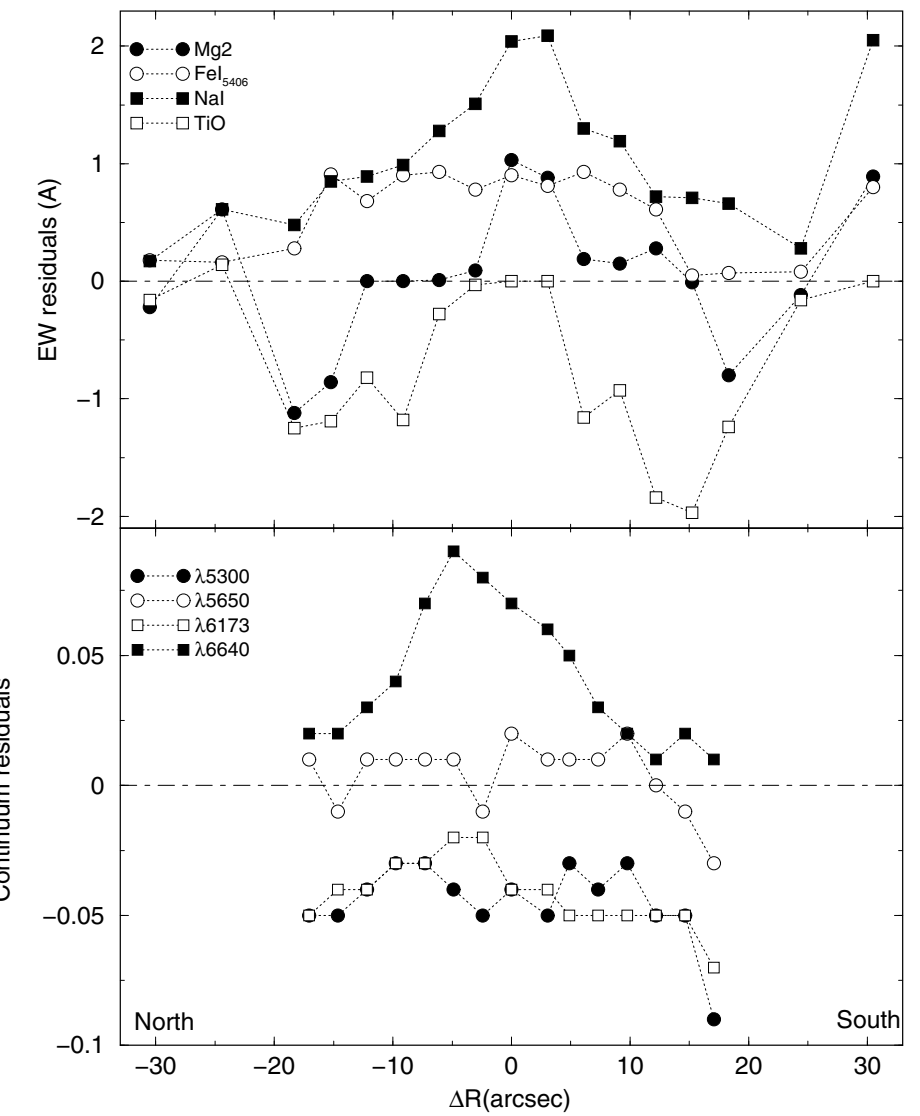

Fig. 6. Stellar population synthesis residuals $\left(E W_{\lambda}(\mathrm{obs})-E W_{\lambda}(\right.$ synt $\left.)\right)$ of representative EWs (top panel) and continuum points (bottom). Negative values in the north direction.

synthesis, especially in terms of metallicity. Nevertheless, Fig. 5 indicates that the $13 \mathrm{Gyr}$ population dominates in NGC 3607 , contributing with approximately $50 \%$ of the flux at $5870 \AA$. The 5 Gyr population contributes with approximately $30 \%$.

Another way to assess the efficiency of the stellar population synthesis algorithm is by means of the residuals (difference between the observed and synthesised values), both in EWs and continuum points. With a few exceptions, the synthesised EWs are within $\pm 1 \AA$ of the observed values, while for the synthesised continuum points (normalised to $\lambda 5870 \AA$ ) the offset lies within \pm 0.1 . We illustrate this in Fig. 6 for representative features and continuum points. The residuals are almost equally distributed between positive and negative values, which indicates that metallicity variations of NGC 3607 are essentially contemplated by the adopted templates (Table 3 ).

\section{Metallicity}

We also investigated the metallicity properties of NGC 3607 by comparing the measured Lick indices of $\mathrm{Mg} 2, \mathrm{Fe}_{\lambda 5270}$, and Fe $\mathrm{I}_{\lambda 5335}$ (Table 2), with the equivalent ones derived from singleaged stellar population (SSP) models (Thomas et al. 2003; Buzzoni et al. 1992) that assume a Salpeter (1955) initial mass function and different ages. After testing a range of SSP ages, we found that the indices measured in NGC 3607 are best described by the 10 Gyr models.

In Fig. 7 we compare our Lick indices measured in NGC 3607 with the equivalent ones computed from SSP models built for different metallicities and $[\alpha / \mathrm{Fe}]$ ratios. The central 


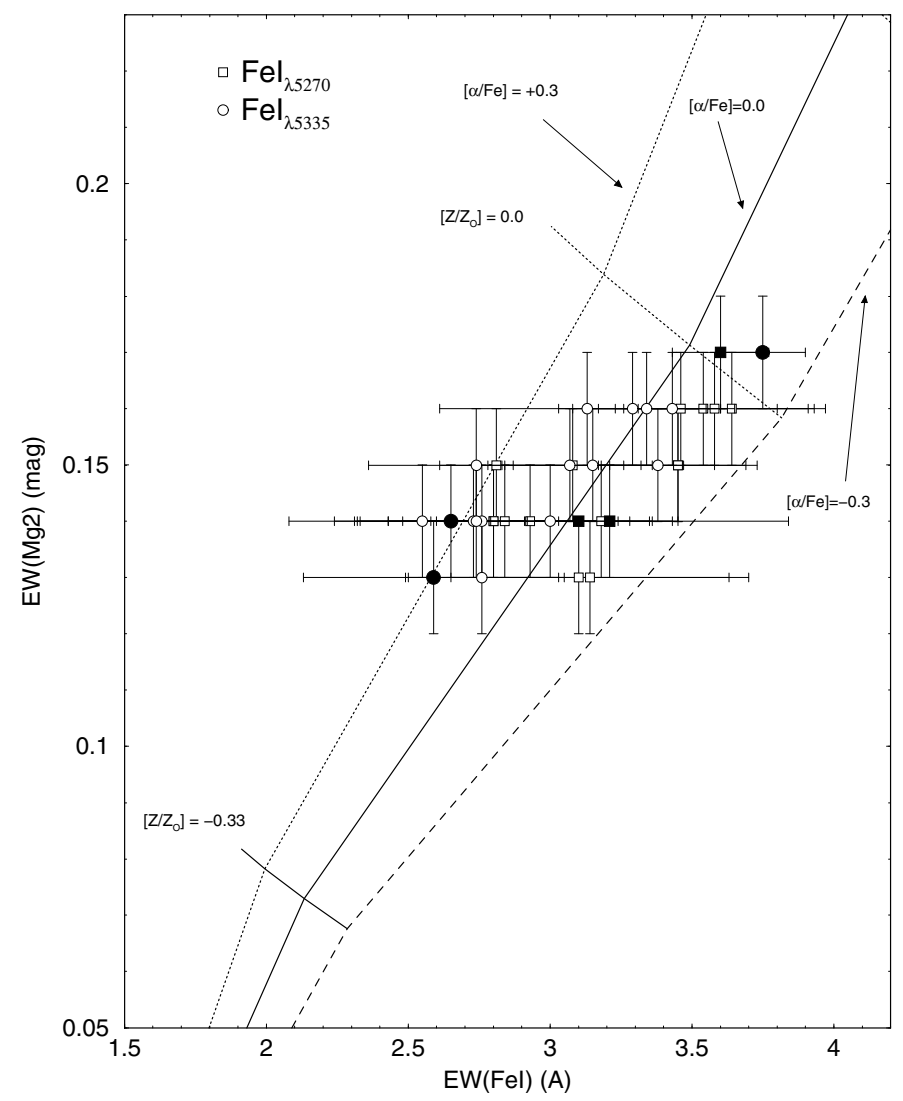

Fig. 7. Selected Lick indices measured in NGC 3607 compared to those computed from SSP models for a range of metallicity and $[\alpha / \mathrm{Fe}]$ ratios. Filled symbols indicate the central (higher values) and external regions (lower values), respectively.

parts of NGC 3607 present a small deficiency of alpha elements with respect to iron $(-0.3 \lesssim[\alpha / \mathrm{Fe}] \lessgtr 0.0)$, whereas the opposite trend appears to characterise the external parts. We find the average value of $[\alpha / \mathrm{Fe}]=0.13$, which is comparable to the value 0.24 reported by Annibali et al. (2007).

NGC 3607 presents sub solar metallicity, with the external parts more metal-poor than the nucleus. The differences observed in $[\alpha / \mathrm{Fe}]$ imply different mechanisms of chemical enrichment for the central and external regions of NGC 3607.

\section{Ionised gas}

Emission gas has been detected in a large number of elliptical and lenticular galaxies (Phillips et al. 1986; Macchetto et al. 1996; Rickes et al. 2004, 2008). However, the origin of this gas and the ionisation source have not yet been conclusively established. It is clear from Fig. 2 that NGC 3607 presents conspicuous emission lines throughout its body. In this section we investigate the physical conditions and ionisation source of the gas.

The properties of the emission gas were derived from line fluxes measured in the stellar population subtracted spectra (Sect. 4). The emission line fluxes of $\mathrm{H} \alpha$, [N II $]_{\lambda 6548},[\mathrm{~N} \mathrm{II}]_{\lambda 6584}$, $[\mathrm{S} \mathrm{II}]_{\lambda 6717}$, and $[\mathrm{S} \mathrm{III}]_{\lambda 6731}$ were measured with Gaussian functions fitted to the profiles. They are listed in Table 4

The spatial distributions of the measured ratios [NII]/H $\alpha$ and [SII]/H $\alpha$ are shown in Fig. 8. While the former presents significant changes with distance to the centre, the latter is more uniformly distributed.

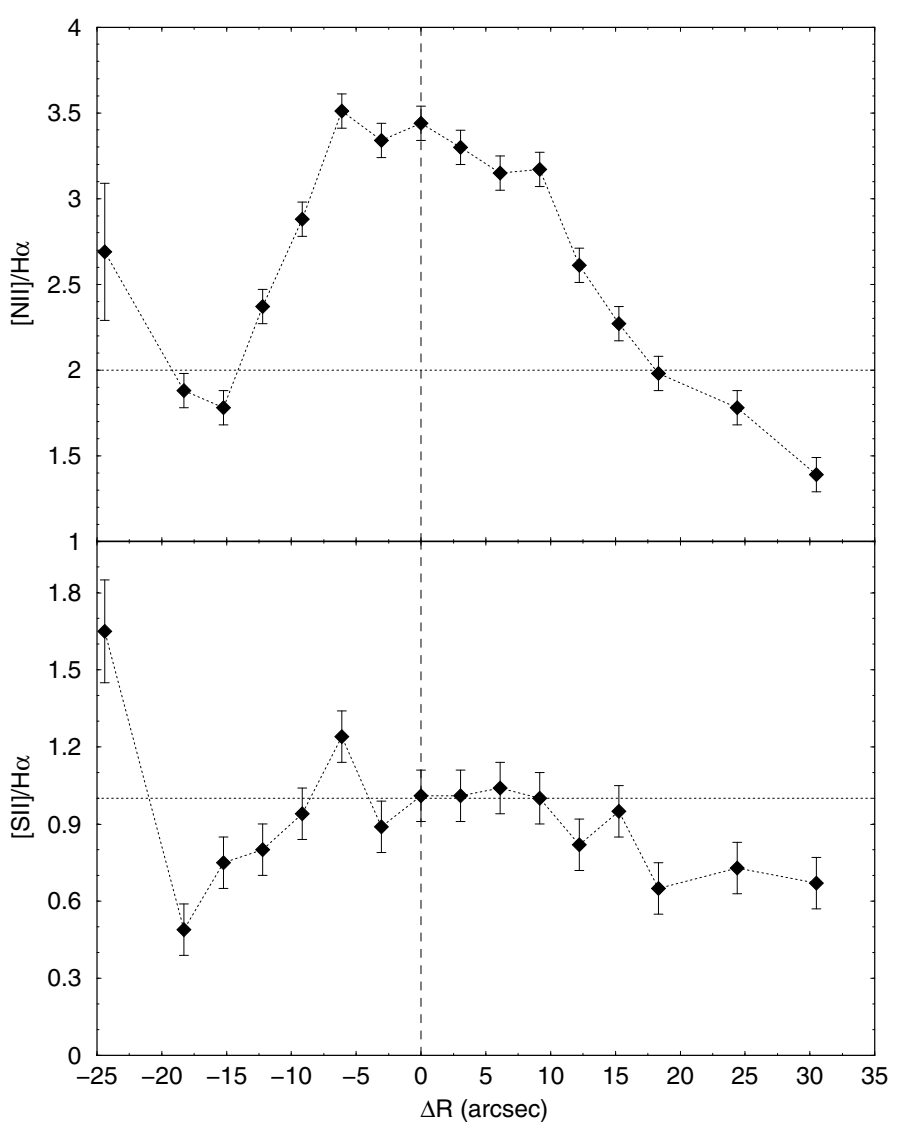

Fig. 8. Spatial variation of the ratios $\frac{[\mathrm{NII}]_{\lambda 6584}}{\mathrm{H} \alpha}$ a) and $\frac{[\mathrm{SII}]_{66731}}{\mathrm{H} \alpha}$ b). Negative values in the north direction.

Assuming that the emission lines are formed essentially by recombination, the number of ionising photons $(Q(\mathrm{H}))$ can be calculated as $Q(\mathrm{H})=\frac{L_{\mathrm{H} \alpha}}{h v_{\alpha}} \frac{\alpha_{B}\left(\mathrm{H}^{0}, T\right)}{\alpha_{\mathrm{H} \alpha}\left(\mathrm{H}^{0}, T\right)}$ (Osterbrock 1989), where $\alpha_{B}\left(\mathrm{H}^{0}, T\right)$ is the total recombination coefficient, and $\alpha_{\mathrm{H} \alpha}\left(\mathrm{H}^{0}, T\right)$ is the recombination coefficient for $\mathrm{H} \alpha$. The values of $Q(\mathrm{H})$ and $L_{\mathrm{H} \alpha}$ are given in Table 5, and their spatial distributions are shown in panels (a) and (b) of Fig. 9. The central region of NGC 3607 presents $[\mathrm{NII}] / \mathrm{H} \alpha=3.44$ and $\left[\mathrm{S}_{\mathrm{II}}\right] / \mathrm{H} \alpha>1$. We note that the ratio $[\mathrm{NII}] / \mathrm{H} \alpha$ is higher than 1.0 for all sampled regions (panel (a)).

Three hypotheses for the nature of the ionising source in the external regions of NGC 3607 are tested: the gas is ionised by (i) an H II region; (ii) hot, post-AGB stars; or (iii) an AGN. They are discussed individually below.

\subsection{HII region}

We build an HII region with a mass distribution that follows the initial mass function of Salpeter (1955), for stellar masses in the range $0.1-100 M_{\odot}$. The model that reproduces the number of ionising photons measured in the region at 3.05" best is described in Table 6. This table provides the distribution of stars for a representative set of spectral type and mass; in the last column we give the number of ionising photons $\left(N_{\mathrm{Ly} \alpha}\right)$ produced by each star (Dottori 1981). The total number of ionising photons can be computed from $N(\mathrm{Ly})=\int_{10}^{30} N_{\mathrm{Ly}}(m) \phi(m) \mathrm{d} m$. The relation between the number of ionising photons with stellar mass can be approximated as $N_{\mathrm{Ly}}(m) \approx 3.9 \times 10^{39}\left(\frac{m}{M_{\odot}}\right)^{5.9} \mathrm{~s}^{-1}$ (Rickes et al. 2008). Given the above, the adopted model H II region 
Table 4. Emission line parameters of NGC 3607.

\begin{tabular}{lccccccc}
\hline \hline$R\left({ }^{\prime \prime}\right)$ & $\mathrm{H} \alpha$ & {$\left[\mathrm{NII}_{6584}\right]$} & {$\left[\mathrm{NII}_{6548}\right]$} & {$\left[\mathrm{SII}_{6717}\right]$} & {$\left[\mathrm{SII}_{6731}\right]$} & $\frac{[\mathrm{INII}]}{\mathrm{H} \alpha}$ & $\frac{[\mathrm{SII}]}{\mathrm{H} \alpha}$ \\
$(1)$ & $(2)$ & $(3)$ & $(4)$ & $(5)$ & $(6)$ & $(7)$ & $(8)$ \\
\hline 0.00 & $1.34 \pm 0.1$ & $3.46 \pm 0.1$ & $1.15 \pm 0.1$ & $0.66 \pm 0.1$ & $0.69 \pm 0.1$ & $3.44 \pm 0.1$ & $1.01 \pm 0.1$ \\
$3.05 \mathrm{~S}$ & $1.29 \pm 0.1$ & $3.20 \pm 0.1$ & $1.06 \pm 0.1$ & $0.58 \pm 0.2$ & $0.72 \pm 0.2$ & $3.30 \pm 0.1$ & $1.01 \pm 0.1$ \\
$6.10 \mathrm{~S}$ & $0.93 \pm 0.1$ & $2.20 \pm 0.1$ & $0.73 \pm 0.1$ & $0.47 \pm 0.2$ & $0.50 \pm 0.2$ & $3.15 \pm 0.1$ & $1.04 \pm 0.1$ \\
$9.15 \mathrm{~S}$ & $0.60 \pm 0.2$ & $1.43 \pm 0.2$ & $0.47 \pm 0.2$ & $0.32 \pm 0.2$ & $0.28 \pm 0.2$ & $3.17 \pm 0.1$ & $1.00 \pm 0.1$ \\
$12.20 \mathrm{~S}$ & $0.56 \pm 0.2$ & $1.10 \pm 0.2$ & $0.36 \pm 0.2$ & $0.14 \pm 0.1$ & $0.32 \pm 0.1$ & $2.61 \pm 0.1$ & $0.82 \pm 0.1$ \\
$15.25 \mathrm{~S}$ & $0.60 \pm 0.2$ & $1.02 \pm 0.2$ & $0.34 \pm 0.2$ & $0.22 \pm 0.2$ & $0.35 \pm 0.2$ & $2.27 \pm 0.1$ & $0.95 \pm 0.1$ \\
$18.30 \mathrm{~S}$ & $0.84 \pm 0.2$ & $1.25 \pm 0.2$ & $0.41 \pm 0.2$ & $0.19 \pm 0.2$ & $0.36 \pm 0.2$ & $1.98 \pm 0.1$ & $0.65 \pm 0.1$ \\
$24.40 \mathrm{~S}$ & $0.80 \pm 0.2$ & $1.07 \pm 0.3$ & $0.35 \pm 0.2$ & $0.28 \pm 0.2$ & $0.30 \pm 0.2$ & $1.78 \pm 0.1$ & $0.73 \pm 0.1$ \\
$30.50 \mathrm{~S}$ & $0.85 \pm 0.3$ & $0.89 \pm 0.3$ & $0.29 \pm 0.3$ & $0.26 \pm 0.2$ & $0.31 \pm 0.2$ & $1.39 \pm 0.1$ & $0.67 \pm 0.1$ \\
\hline $3.05 \mathrm{~N}$ & $0.97 \pm 0.1$ & $2.43 \pm 0.1$ & $0.81 \pm 0.1$ & $0.42 \pm 0.2$ & $0.44 \pm 0.2$ & $3.34 \pm 0.1$ & $0.89 \pm 0.1$ \\
$6.10 \mathrm{~N}$ & $0.71 \pm 0.1$ & $1.87 \pm 0.1$ & $0.62 \pm 0.1$ & $0.41 \pm 0.2$ & $0.47 \pm 0.2$ & $3.51 \pm 0.1$ & $1.24 \pm 0.1$ \\
$9.15 \mathrm{~N}$ & $0.66 \pm 0.2$ & $1.43 \pm 0.2$ & $0.47 \pm 0.2$ & $0.31 \pm 0.2$ & $0.31 \pm 0.2$ & $2.88 \pm 0.1$ & $0.94 \pm 0.1$ \\
$12.20 \mathrm{~N}$ & $0.65 \pm 0.2$ & $1.16 \pm 0.2$ & $0.38 \pm 0.2$ & $0.26 \pm 0.2$ & $0.26 \pm 0.2$ & $2.37 \pm 0.1$ & $0.80 \pm 0.1$ \\
$15.25 \mathrm{~N}$ & $0.80 \pm 0.2$ & $1.07 \pm 0.3$ & $0.35 \pm 0.2$ & $0.30 \pm 0.2$ & $0.30 \pm 0.2$ & $1.78 \pm 0.1$ & $0.75 \pm 0.1$ \\
$18.30 \mathrm{~N}$ & $0.90 \pm 0.3$ & $1.27 \pm 0.3$ & $0.42 \pm 0.3$ & $0.22 \pm 0.3$ & $0.22 \pm 0.3$ & $1.88 \pm 0.1$ & $0.49 \pm 0.1$ \\
$24.40 \mathrm{~N}$ & $0.51 \pm 0.4$ & $1.03 \pm 0.4$ & $0.34 \pm 0.4$ & $0.42 \pm 0.3$ & $0.42 \pm 0.3$ & $2.69 \pm 0.4$ & $1.65 \pm 0.2$ \\
\hline
\end{tabular}

Notes. $\mathrm{H} \alpha$ flux in Col. 2 is given in $10^{-15} \mathrm{erg} \mathrm{s}^{-1} \mathrm{~cm}^{-2}$. Column 7: [NII] $]_{\lambda 6584+6548}$; Col. 8: [SII $]_{\lambda 6717+6731}$.

Table 5. H $\alpha$ luminosity and number of ionising photons.

\begin{tabular}{lccc}
\hline \hline$R$ & $F_{\mathrm{H} \alpha}$ & $\begin{array}{c}L_{\mathrm{H} \alpha} \\
\left({ }^{\prime \prime}\right)\end{array}$ & $\begin{array}{c}Q(\mathrm{H}) \\
\left(10^{-15} \mathrm{erg} \mathrm{cm}^{-2} \mathrm{~s}^{-1}\right)\end{array}$ \\
\hline $0.00 \mathrm{~S}$ & $1.34 \pm 0.10$ & $2.61 \pm 0.03$ & $13.7 \pm 0.02$ \\
$3.05 \mathrm{~S}$ & $1.29 \pm 0.10$ & $2.51 \pm 0.02$ & $13.2 \pm 0.02$ \\
$6.10 \mathrm{~S}$ & $0.93 \pm 0.01$ & $1.81 \pm 0.03$ & $9.54 \pm 0.01$ \\
$9.15 \mathrm{~S}$ & $0.60 \pm 0.02$ & $1.17 \pm 0.02$ & $6.15 \pm 0.02$ \\
$12.20 \mathrm{~S}$ & $0.56 \pm 0.02$ & $1.09 \pm 0.02$ & $5.74 \pm 0.03$ \\
$15.25 \mathrm{~S}$ & $0.60 \pm 0.02$ & $1.17 \pm 0.03$ & $6.15 \pm 0.03$ \\
$18.30 \mathrm{~S}$ & $0.84 \pm 0.02$ & $1.63 \pm 0.02$ & $8.62 \pm 0.03$ \\
$24.40 \mathrm{~S}$ & $0.80 \pm 0.02$ & $1.56 \pm 0.02$ & $8.21 \pm 0.01$ \\
$30.50 \mathrm{~S}$ & $0.85 \pm 0.03$ & $1.65 \pm 0.02$ & $8.72 \pm 0.02$ \\
\hline $3.05 \mathrm{~N}$ & $0.97 \pm 0.01$ & $1.89 \pm 0.03$ & $9.95 \pm 0.02$ \\
$6.10 \mathrm{~N}$ & $0.71 \pm 0.01$ & $1.38 \pm 0.03$ & $7.28 \pm 0.01$ \\
$9.15 \mathrm{~N}$ & $0.66 \pm 0.02$ & $1.28 \pm 0.03$ & $6.77 \pm 0.01$ \\
$12.20 \mathrm{~N}$ & $0.65 \pm 0.02$ & $1.26 \pm 0.03$ & $6.67 \pm 0.02$ \\
$15.25 \mathrm{~N}$ & $0.80 \pm 0.02$ & $1.56 \pm 0.03$ & $8.21 \pm 0.02$ \\
$18.30 \mathrm{~N}$ & $0.90 \pm 0.03$ & $1.75 \pm 0.03$ & $9.23 \pm 0.02$ \\
$24.40 \mathrm{~N}$ & $0.51 \pm 0.04$ & $0.99 \pm 0.03$ & $5.23 \pm 0.01$ \\
\hline
\end{tabular}

Notes. H $\alpha$ luminosity and number of ionising photons computed for the extractions given in Col. 1.

produces $\sim 2.6 \times 10^{50}$ photons $\mathrm{s}^{-1}$, which is comparable to the observed value. However, with more than $20 \mathrm{O}$ stars, such an H II region would be very young, massive, and luminous enough to have already been detected in previous works. Besides, the stellar population synthesis (Sect. 4) does not indicate significant, recent star formation in the sampled regions of NGC 3607.

\subsection{Post-AGB stars}

The number of ionising photons emitted by a typical post-AGB star is $\sim 10^{47} \mathrm{~s}^{-1}$ (Binette 1994). Thus, about $10^{4}$ such stars would be necessary to produce the amount of photons measured

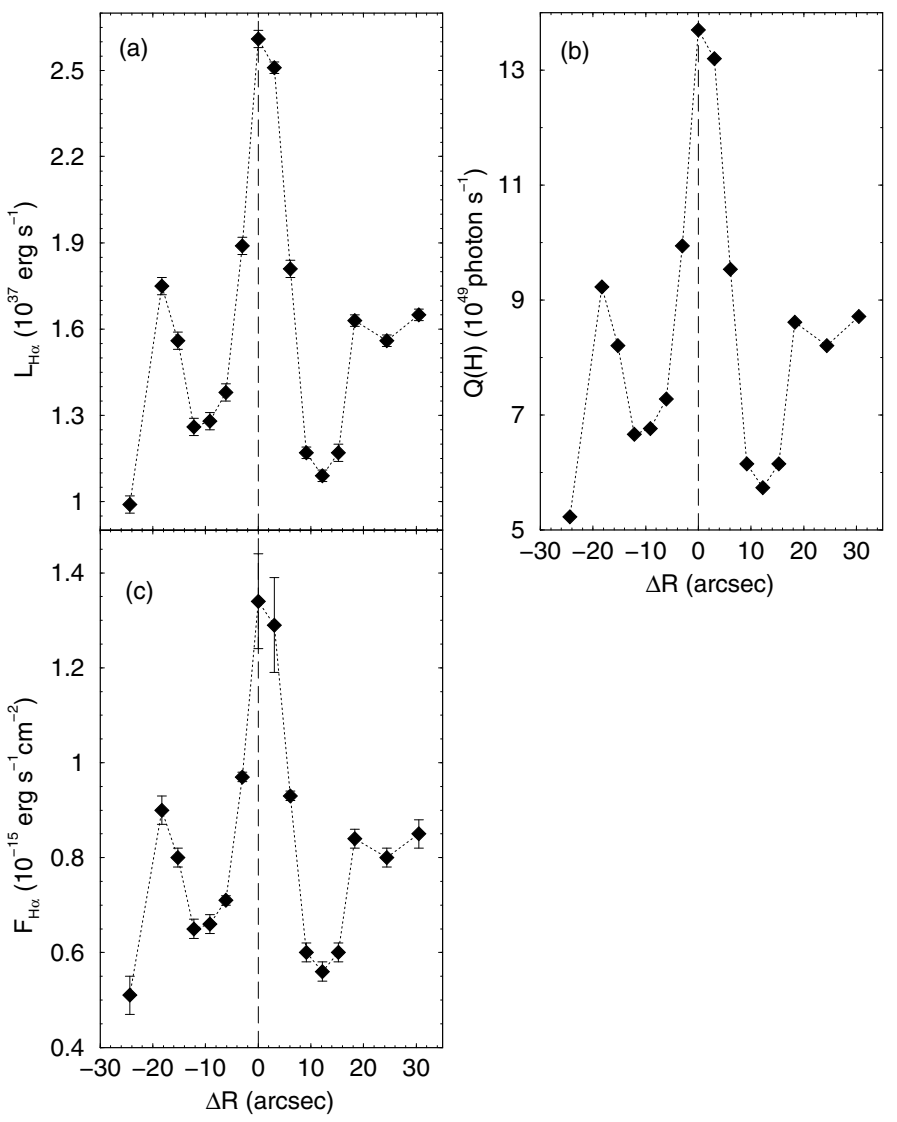

Fig. 9. Spatial distribution of the $\mathrm{H} \alpha$ luminosity a) flux c) and number of ionising photons b). Negative values in the north direction.

in each extracted region. Consequently, for an extraction area of $\approx 0.02 \mathrm{kpc}^{2}$ (Sect. 2), the projected surface-density of post-AGB stars would be about $5 \times 10^{5} \mathrm{kpc}^{-2}$, a prohibitively high number (e.g. Binette 1994). Besides, the spatial profiles of [N II] and $\mathrm{H} \alpha$ extracted along the slit (Fig. 8) show a ratio $\mathrm{N}[\mathrm{II}] / \mathrm{H} \alpha>2$ 
Table 6. H II region model parameters.

\begin{tabular}{lccc}
\hline \hline Spectral type & $\begin{array}{c}M \\
\left(M_{\odot}\right)\end{array}$ & $\begin{array}{c}\text { Number } \\
(\text { stars })\end{array}$ & $\begin{array}{c}N_{\mathrm{Ly} \alpha} \\
\left(10^{48} \text { photon s }^{-1}\right)\end{array}$ \\
\hline O5 & 30 & 20 & 2.00 \\
& 22 & 41 & 0.25 \\
B0 & 17 & 75 & 0.082 \\
& 15 & 101 & 0.043 \\
& 13 & 142 & 0.015 \\
& 10 & 264 & 0.0021 \\
\hline
\end{tabular}

Notes. Column 2: representative stellar mass; Col. 3: number of stars; Col. 3: number of ionising photons.

for most regions, especially towards the central parts. However, the relatively high emission-line ratios measured in NGC 3607 , especially in the central region, are not consistent with ionisation models based on hot old stars in elliptical galaxies (e.g. Binette 1994). The models of Binette (1994) do not reproduce the observed high ratios $[\mathrm{N} \mathrm{II}] / \mathrm{H} \alpha$ and $[\mathrm{S} \mathrm{II}]_{6731} / \mathrm{H} \alpha$ as well. Instead, high values for these emission-line ratios are typical of AGNs. On the other hand, the lower ratios of $[\mathrm{NII}]_{\lambda 6584} / \mathrm{H} \alpha$ and $[\mathrm{SII}]_{6731} / \mathrm{H} \alpha$ (Fig. 8), together with the lower values of $Q(\mathrm{H})$ (Fig. 9) measured for large radii, may, in part, be accounted for by hot old stars.

\subsection{Active nucleus}

The arguments that follow from the above discussion suggest that NGC 3607 hosts an AGN. We test this hypothesis with the emission-line diagnostic diagram involving the ratios $[\mathrm{NII}]_{\lambda 6584} / \mathrm{H} \alpha$ and $[\mathrm{SII}]_{\lambda 6717,31} / \mathrm{H} \alpha$. For comparison we take the equivalent values measured in a sample of nearby galaxies that host a low-luminosity $\left(L_{\mathrm{H} \alpha}<2 \times 10^{39} \mathrm{erg} \mathrm{s}^{-1}\right)$ active nucleus (Ho et al. 1997). To minimise the possibility of uncertain classification, we restrict the analysis to the galaxies in the Ho et al. (1997) sample with unambiguous classification as LINER in spiral, LINER in elliptical, Seyfert, or starburst. The restricted sample contains 128 galaxies (see Rickes et al. 2008).

The results are shown in Fig. 10, where it can be seen that the values measured in NGC 3607 occur in the locus occupied predominantly by LINERs and Seyferts. However, the lack of a broad component in $\mathrm{H} \alpha$ (Fig. 2) strongly favours the LINER interpretation. Together with the stellar population synthesis, this result suggests that the main source of gas ionisation in NGC 3607 is non thermal, produced by a low-luminosity active nucleus. The lower ratios of $[\mathrm{NII}]_{\lambda 6584} / \mathrm{H} \alpha$ and $[\mathrm{SII}]_{6731} / \mathrm{H} \alpha$ towards the external parts can be accounted for by both a LINER and hot stars.

Finally, in Fig. 11 we test whether the gradients in emission and absorption-line features are somehow related. We work with the $E W$ of $\mathrm{Mg}_{2} \lambda 5176$ (Table 2) and the $\mathrm{H} \alpha$ flux (Table 4) distributions, taking the average values of the north and south extractions. The 2 outermost points have been averaged out to minimise errors. Within the uncertainties, both distributions appear to correlate for $R \lesssim 12^{\prime \prime}$, which can account for the steep and monotonic $\mathrm{H} \alpha$ flux increase towards the central region (Fig. 9), and the overall negative $\mathrm{Mg} 2$ gradient (Fig. 3). While the $E W$ of $\mathrm{Mg}_{2}$ keeps dropping for $R \gtrsim 12^{\prime \prime}$, the $\mathrm{H} \alpha$ flux oscillates abruptly, probably because of the relative contribution of the old hot stars to the gas ionisation, superimposed on the severely diluted LINER flux at such large radii. This combination of effects is reflected in the mild anti-correlation of both properties (lower

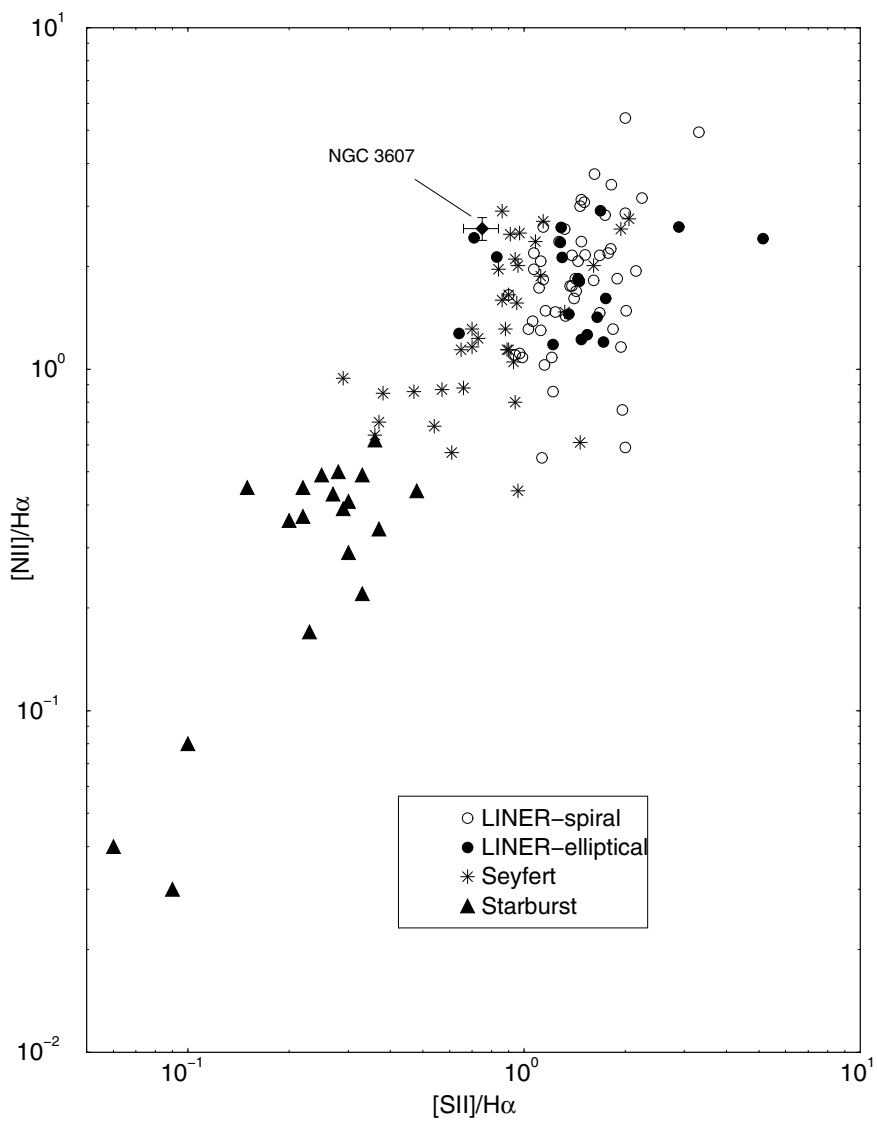

Fig. 10. Emission-line diagnostic diagrams with a subsample of the galaxies in Ho et al. (1997) plotted as comparison. The locus of NGC 3607 is consistent with that of LINERs.

portion of Fig. 11). It is also consistent with the low ratios of $[\mathrm{N} \mathrm{II}] / \mathrm{H} \alpha$ and [S II]/H $\alpha$ (Fig. 8), and the low $Q(\mathrm{H})$ (Fig. 9), measured for large radii, all accounted for by old hot stars.

Similar results would be obtained with other absorption features, since they also present similar gradients (Fig. 3).

\section{Discussion and concluding remarks}

Individual galaxies can provide important pieces of evidence that may ultimately lead to better understanding the formation and evolution processes of the early-type galaxies as a class. Following the methods previously employed in analysing other early-type galaxies (e.g. Rickes et al. 2004; Rickes et al. 2004), we focussed on the lenticular galaxy NGC 3607.

We investigated the stellar population, metallicity distribution, and ionised gas of NGC 3607 by means of long-slit spectroscopy (covering the wavelength range $\lambda \lambda 5100-6800 \AA$ ) and a previously tested stellar population synthesis algorithm. The stellar population synthesis shows different star formation episodes with ages distributed within 1-13 Gyr.

The radial distribution of the equivalent widths measured for the available metallic absorption features in this galaxy consistently present a negative gradient, which indicates an overabundance of $\mathrm{Fe}, \mathrm{Mg}, \mathrm{Na}$, and $\mathrm{TiO}$ in the central parts with respect to the external regions. Also, the central region of NGC 3607 presents more of a deficiency of alpha elements and higher metallicity than do the external parts, which suggests different star formation histories in both regions. With respect to the emission gas, the line ratios $[\mathrm{NII}]_{\lambda 6584} / \mathrm{H} \alpha$ and $[\mathrm{SII}]_{\lambda 6717,31} / \mathrm{H} \alpha$ 


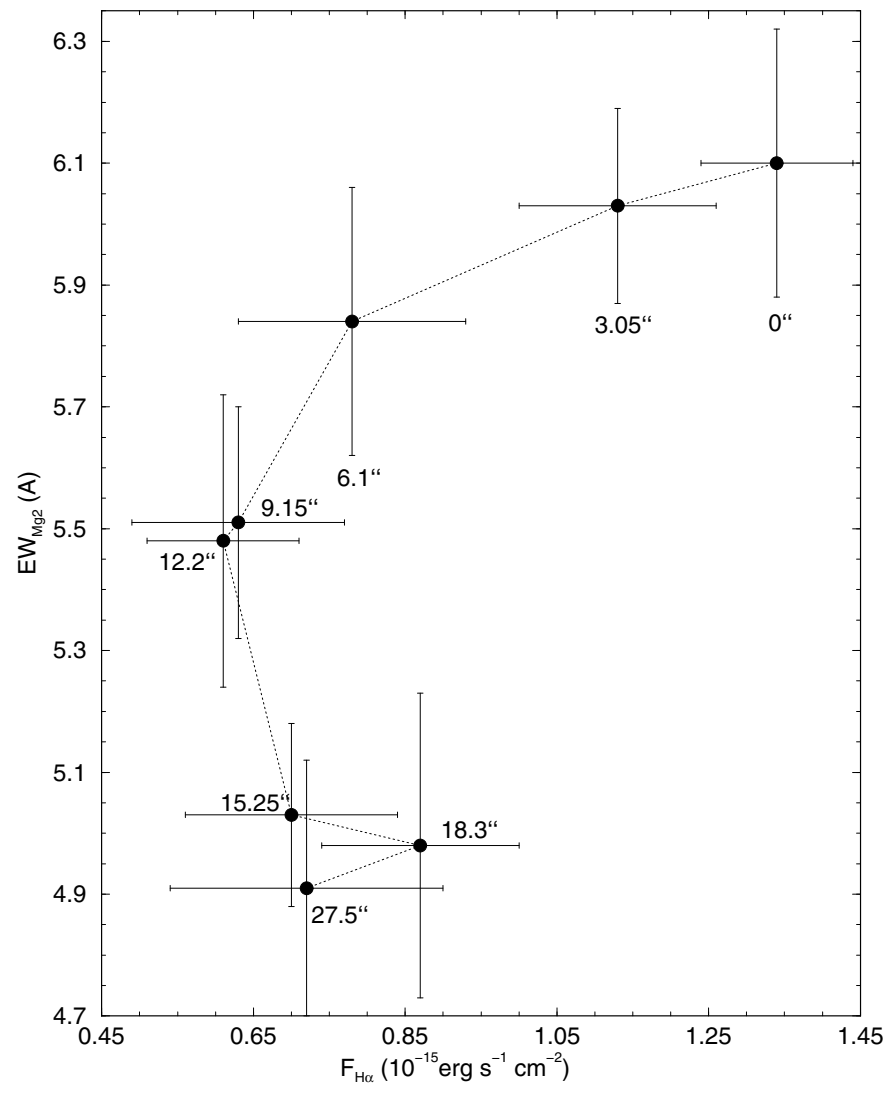

Fig. 11. Relation between $E W\left(\mathrm{Mg}_{2}\right.$ 15176) and the $\mathrm{H} \alpha$ flux as a function of distance to the galaxy centre.

consistently indicate the presence of a LINER at the centre of NGC 3607.

We found that the $\mathrm{Mg}_{2}$ index correlates both with $\mathrm{FeI}_{\lambda 5270}$ and $\mathrm{FeI}_{\lambda 5335}$, which suggests that $\mathrm{Mg}$ and $\mathrm{Fe}$ probably underwent the same enrichment process in NGC 3607. Also, the $\mathrm{H} \alpha$ flux distribution correlates with the radial profile of metallic features, in the sense that they both decrease from the galaxy centre to $R \approx 12^{\prime \prime}$. The correlation breaks for larger radii, especially because, while the metallic feature profiles keep dropping, gas ionisation from old hot stars adds to the diluted LINER flux. Considering the relation between galaxy dynamical mass and the $M g_{2}$ gradient slope (Fig. 11 of Carollo et al. 1993), the parameters computed for NGC 3607 in the present work suggest that this galaxy may have undergone major merger events in the past.

Acknowledgements. We acknowledge important suggestions presented by the referee. We thank the Brazilian agency $\mathrm{CNPq}$ for partial support of this work. This research made use of the NASA/IPAC Extragalactic Database (NED), which is operated by the Jet Propulsion Laboratory, California Institute of Technology, under contract with the National Aeronautics and Space Administration.

\section{References}

Annibali, F., Bressan, A., Rampazzo, R., Zeilinger, W. W., \& Danese, L. 2007, A\&A, 463, 455

Arimoto, N., \& Yoshii, Y. 1987, A\&A, 23, 38

van den Bergh, S. 1990, MNRAS, 31, 153

Bica, E., \& Alloin, D. 1986, A\&AS, 66, 171

Bica, E. 1988, A\&A, 195, 79

Binette, L. Magris, C. G. Stasinska, G., \& Bruzual, A. G. 1994, A\&A, 292, 13

Bruzual, G., \& Charlot, S. 2003, MNRAS, 344, 1000

Buzzoni, A., Mantegazza, L., \& Gariboldi, G. 1994, AJ., 107, 513

Caon, N., Macchetto, D., \& Pastoriza, M. 2000, ApJS, 127, 39

Carollo, C. M., \& Danziger, I. J. 1994, MNRAS, 270, 523

Carollo, C. M., Danziger, I. J., \& Buson, L. 1993, MNRAS, 265, 553

Davies, R. L., Sadler, E. M., \& Peletier, R. F. 1993, MNRAS, 262, 650

Dottori, H. A. 1981, ApSS, 80, 267

Faber, S. M., Friel, E. D., Burstein, D., \& Gaskell, C. M. 1985, ApJS, 57, 711

Ferguson, H., \& Sandage, A. 1990, AJ, 100, 1

Ferrari, F., Pastoriza, M. G., Macchetto, F., \& Caon, N. 1999, A\&A, 136, 269

Fisher, D., Franx, M., \& Illingworth, G. 1995, ApJ, 448, 119

Fisher, D., Franx, M., \& Illingworth, G. 1996, ApJ, 459, 110

Gonzáles, J. J. 1993, Ph.D. Thesis, Univ. California, Santa Cruz

Idiart, T. P., Michard, R., \& de Freitas Pacheco, J. A. 2003, A\&A, 398, 949

Ho, L. C., Filippenko, A. V., \& Sargent, W. L. W. 1997, ApJS, 112, 315

Larson, Richard B. 1974, MNRAS, 166, 585

Maia, M. A. G., Da Costa, L. N., \& Latham, D. W., 1989, AJSS, 69, 809

Macchetto, F., Pastoriza, M. G., Caon, N., et al. 1996, ApJS, 120, 463

Matsushita, K., Ohashi, T., \& Makishima, K. 2000, PASJ, 52, 685

Ogando, R. L. C., Maia, M. A. G., Chiappini, C., et al. 2005, ApJ, 61, 64

Osterbrock, D. E. 1989, in Astrophysics of Gaseous Nebulae and Active Galactic

Nuclei (University Science Books)

Phillips, M. M., Jenkins, C. R., Dopita, M. A., Sadler, E. M., \& Binette, L. 1986, AJ, 91, 1062

Pipino, A., Matteucci, F., \& Chiappini, C. 2006, ApJ, 638, 739

Poveda, A., \& Allen, C. 1975, ApJ, 200, 42

Rickes, M. G., Pastoriza, M. G., \& Bonatto, C. 2004, A\&A, 419, 449

Rickes, M. G., Pastoriza, M. G., \& Bonatto, C. 2008, MNRAS, 384, 1427

Salpeter, E. F. 1979, ApJ, 121, 161

Terashima, Y., Iyomoto, N., Ho, L. C., \& Ptak, A. F. 2002, ApJS, 139, 1

Thomas, D., Maraston, C., \& Bender, R. 2003, MNRAS, 339, 897

Worthey, G., Faber, S. M., \& Gonzáles, J. J. 1992, ApJS, 398, 69 